\title{
Editorial: Astrobiology of Mars, Europa, Titan and Enceladus - Most Likely Places for Alien Life
}

\author{
Isik Kanik ${ }^{1,2 *}$ and Jean-Pierre Paul de Vera $^{3}$ \\ ${ }^{1}$ Jet Propulsion Laboratory, California Institute of Technology, Pasadena, CA, United States, ${ }^{2}$ NASA Astrobiology Institute - Icy \\ Worlds, Pasadena, CA, United States, ${ }^{3}$ German Aerospace Center, Institute of Planetary Research, Berlin, Germany
}

Keywords: astrobiology, Mars, Europa, Titan, Enceladus

\section{Editorial on the Research Topic}

\section{Astrobiology of Mars, Europa, Titan and Enceladus - Most Likely Places for Alien Life}

A primary science goal of astrobiology is to understand the biological potential of Solar System bodies. Looking beyond Earth, four planetary bodies, namely Mars, Europa, Titan and Enceladus, offer numerous opportunities to investigate the chemical evolution and origin of life. These bodies may have biochemistry similar to that of life on Earth (Davila and McKay, 2014; Taubner et al., 2018; Taubner et al., 2020). In recent years, exploration strategies have been developed to pursue astrobiological objectives for these targets. Two key scientific objectives of current and planned space missions to these bodies are 1) to investigate the abundance and distribution of the organic compounds and biomolecules, as well as environmental conditions (causing alteration, degradation and even complete destruction of these compounds such as irradiation, mineralogy etc.), as these are crucial in the search of biosignatures; and 2) to identify evidence of ancient biota and to determine whether indigenous organisms currently exist anywhere on the surface and subsurface of these planetary bodies.

Because of its many Earth-like features, Mars remains the most intriguing of the planets and has become the preeminent target of astrobiological interest. While Mars today is a cold and dry planet, conditions in the past are thought to have been more benign (McKay and Davis, 1991), and included habitable environments compatible with terrestrial life (Grotzinger et al., 2014). Searching for biogenic elements and organic compounds, and understanding their interactions with the environment will be an important task for the various missions to Mars such as Mars 2020, ExoMars and others that are anticipated over the next few decades by major space agencies such as NASA and the European Space Agency.

More recently, however, two ice covered ocean worlds, Europa and Enceladus, have become increasingly attractive targets for space agencies not only for their biological potential for harboring extant/extinct life but also for being plausible models for the emergence of life independent of Earth evolution. Two successive flagship missions by NASA to Europa (Europa Clipper and Europa Lander) are expected to be launched within the next decade to find evidence of life on an iced covered ocean world. This would be the first life detection mission since the Viking missions on Mars in the 1970s.

In the case of Enceladus, the Cassini spacecraft measurements have already provided sufficient evidence regarding the habitability of Enceladus' ocean which makes it high priority candidate for a search-for-life mission at Enceladus in the next decade. Evidence for an ongoing hydrothermal activity within Enceladus (Hsu et al., 2015; Choblet et al., 2017) and potential hydrothermal activity within Europa (Lunine, 2017), similar to those found on Earth's Atlantic Ocean floor (Kelly et al., 2001; Postberg, et al., 2018) strengthens the arguments regarding their potential to harbor past or present life within their oceans. 
The Cassini-Huygens mission has shown that Titan, the only moon known to have a dense atmosphere, is an unusual world in our Solar System. Although it is far from the Sun and hence cold, solar radiation interacts with its methane rich atmosphere instigating the formation of complex organic molecules and erosols that eventually deposit on Titan's surface, where further chemical reactions could take place. This may be leading to greater molecular complexity and eventually to the most complex organic building blocks of life. In addition, recent Cassini gravity data suggest that Titan's global subsurface ocean may be in contact with the rock core, potentially providing redox gradients and heavier elements critical for a habitable environment. In addition, at the surface, liquid water delivered from Titan's ocean through cryovolcanic activity could indicate the presence of habitable environments if not on the surface because of the extremely cold surface temperatures, so in the subsurface close to the surface.

In this Research Topic on Astrobiology of Mars, Europa, Titan and Enceladus, our aim is to emphasize the importance of astrobiology studies of our own cosmic backyard, highlighting the Solar System bodies where we might find alien life beyond Earth.

Marín-Yaseli et al. investigated reactivity of Cyanide at WaterIce Interfaces since Cyanide has been proposed that polymerization of $\mathrm{NH}_{4} \mathrm{CN}$ would be applicable to icy planetary environments. They, as an approach to understand the possible organic chemistry of icy worlds, explored the effect of water freeze-thaw cycles, UVradiation and salts on the polymerization of $\mathrm{NH}_{4} \mathrm{CN}$. They synthesized insoluble and soluble $\mathrm{NH}_{4} \mathrm{CN}$ polymers, under multiple conditions, and analyzed by GC-MS. Their analysis resulted in identification of a diverse set of amino acids, carboxylic acids, and several N-heterocycles. Utilizing a multivariate analysis technique to their GC-MS results indicated that the ice-water interfaces may be ideal places to develop complex organic chemistry from a carbon source as simple as cyanide.

Pasek conducted a modeling study investigating plume sample modification during the ejection and its implications for biosignatures at Icy Moons such as Europa and Enceladus relevant to in situ direct sampling (with potential for sample return) of liquid water from these bodies. This is critical to determine how representative the collected plume samples are of their environment, determining biosignatures and habitability. His results indicate that water droplets comprising part of plume are frozen rapidly, with loss of about $17 \%$ of the water by evaporation and sublimation if the water is originally liquid and at $273 \mathrm{~K}$. The $17 \%$ loss of water corresponds to a $20 \%$ increase in the concentration of non-volatile solutes such as salts, if the associated water vapor is not collected. Volatiles, such as gases and clathrates (Mousis et al., 2013), will be altered during sublimation, at least as far as their concentration is concerned. Mitigation of this alteration will be highly critical to successful sample collection mission.

Poggiali et al. conducted a laboratory investigation on ultraviolet photoprocessing of Glycine adsorbed on various space-relevant minerals such as serpentine antigorite, olivine forsterite, oxide mineral spinel, and iron-sulfide mineral pyrite. Being the simplest of the 20 amino acids from which complex organic molecules might have originated in our evolved Solar System, Glycine has a significant importance. Possible changes of glycine functional groups due to UV irradiation through in situ infrared (IR) spectroscopic analysis were investigated. Results show that degradation of glycine occurs with a half-life of $0.5-2 \mathrm{~h}$ depending on the mineral substrate. They highlight a lower efficiency of antigorite and spinel in catalyzing GLY photoreaction compared with forsterite and pyrite. Appearance of new IR bands suggests the occurrence of catalytic reactions mediated by minerals and UV. This investigation suggests that mineral composition is an important factor to be considered for positive detection of biomarkers.

Fornaro et al. carried out a comprehensive investigation comprised of an experimental study combined with anharmonic computational spectroscopy calculations on UV irradiation and near infrared characterization of laboratory Mars soil analog samples. They reported changes of the NIR spectroscopic features due to molecule-mineral interactions, molecular concentration, presence of oxidants such as magnesium perchlorate, and UV irradiation, for molecules relevant in the context of life detection, such as adenosine 5 '-monophosphate, L-glutamic acid, L-phenylalanine, and phthalic acid, adsorbed on montmorillonite, a clay mineral common on Mars. They observed that molecule-mineral interactions and UV irradiation cause the most significant changes in NIR spectra. Their investigation emphasizes the importance of building spectroscopic databases for molecule-mineral complexes under martian-like conditions in order to assist interpretation of data collected by space instruments based on NIR spectroscopy.

Neveu et al. provided a comprehensive review on the evidence on Enceladus' habitability and rationalize the value of sample return for life detection from its plume material. In doing so, they estimate the amount of sample that would have to be returned to Earth in order to perform a suite of measurements to assess the presence of signature of life in the samples. Then they provide a discussion on a range of sample return mission architectures and finally provide an outline on technical and policy related unresolved issues to achieve such a mission. Finally, they conclude their review by placing Enceladus sample return in the broader context of Solar System exploration.

Fagliarone et al., conducted a laboratory investigation on biomarker preservation and survivability under the Mars-Like conditions (i.e., extreme dryness and UV flux) relevant to future life detection missions on Mars. For this study, they used desert Cyanobacterium (anhydrobiotic cyanobacterium Chroococcidiopsis) capable of Trehalose and Sucrose accumulation and investigated its survivability and biomarker preservation by performing an in silico analysis of the genome of the CCMEE 029 strain. This work provides a better understanding for the adaptation strategies to extremely dry conditions and suggests that sucrose and trehalose accumulation might reduce macromolecular susceptibility to chemical and physical degradation taking place after cell death. This result has implications for the designing of future life detection missions to Mars and, generally, for biomarker detectability strategies.

\section{AUTHOR CONTRIBUTIONS}

All authors listed have made a substantial, direct, and intellectual contribution to the work and approved it for publication. 


\section{REFERENCES}

Choblet, G., Tobie, G., Sotin, C., Běhounková, M., Čadek, O., Postberg, F., et al. (2017). Powering prolonged hydrothermal activity inside Enceladus. Nat. Astron. 1, 841-847. doi:10.1038/s41550-017-0289-8

Davila, A. F., and McKay, C. P. (2014). Chance and necessity in biochemistry: implications for the search for extraterrestrial biomarkers in earth-like environments. Astrobiology 14, 534-540. doi:10.1089/ast.2014.1150

Grotzinger, J. P., Sumner, D. Y., Kah, L. C., Stack, K., Gupta, S., Edgar, L., et al. (2014). A habitable fluvio-lacustrine environment at yellowknife bay, gale crater, Mars. Science 343, 1242777. doi:10.1126/science.1242777

Hsu, H. W., Postberg, F., Sekine, Y., Shibuya, T., Kempf, S., Horányi, M., et al. (2015). Ongoing hydrothermal activities within Enceladus. Nature 519, 207-210. doi:10.1038/nature14262

Kelley, D., Karson, J., Blackman, D., Früh-Green, G. L., Butterfield, D. A., Lilley, M. D., et al. (2001). An off-axis hydrothermal vent field near the Mid-Atlantic Ridge at 30 degrees N. Nature 412, 145-149. doi:10.1038/ 35084000

Lunine, J. I. (2017). Ocean worlds exploration. Acta Astronautica 131, 123-130. doi:10.1016/j.actaastro.2016.11.017

McKay, C. P., and Davis, W. L. (1991). Duration of liquid water habitats on early Mars. Icarus 90, 214-221. doi:10.1016/0019-1035(91)90102-Y
Mousis, O., Lakhlifi, A., Picaud, S., Pasek, M., and Chassefière, E. (2013). On the abundances of noble and biologically relevant gases in lake vostok, Antarctica. Astrobiology 13, 380-390. doi:10.1089/ast.2012.0907

Postberg, F., Khawaja, N., Abel, B., Choblet, G., Glein, C. R., Gudipati, M. S., et al. (2018). Macromolecular organic compounds from the depths of Enceladus. Nature 558, 564-568. doi:10.1038/s41586-018-0246-4

Taubner, R. S., Olsson-Francis, K., Vance, S. D., Ramkissoon, N. K., Postberg, F., de Vera, J. P., et al. (2020). Experimental and simulation efforts in the astrobiological exploration of exooceans. Space Sci. Rev. 216, 9. doi:10.1007/s11214-020-0635-5

Taubner, R. S., Pappenreiter, P., Zwicker, J., Smrzka, D., Pruckner, C., Kolar, P., et al. (2018). Biological methane production under putative Enceladus-like conditions. Nat. Commun. 9, 748. doi:10.1038/s41467-018-02876-y

Conflict of Interest: The authors declare that the research was conducted in the absence of any commercial or financial relationships that could be construed as a potential conflict of interest.

Copyright (C) 2021 Kanik and Paul de Vera. This is an open-access article distributed under the terms of the Creative Commons Attribution License (CC BY). The use, distribution or reproduction in other forums is permitted, provided the original author(s) and the copyright owner(s) are credited and that the original publication in this journal is cited, in accordance with accepted academic practice. No use, distribution or reproduction is permitted which does not comply with these terms. 